\title{
Turbulence imaging and applications using beam emission spectroscopy on DIII-D (invited)
}

\author{
G. R. McKee ${ }^{\text {a) }}$ C. Fenzi, ${ }^{\text {b) }}$ R. J. Fonck, and M. Jakubowski \\ University of Wisconsin-Madison, 1500 Engineering Drive, Madison, Wisconsin 53706
}

(Presented on 10 July 2002)

\begin{abstract}
Two-dimensional measurements of density fluctuations are obtained in the radial and poloidal plane of the DIII-D tokamak with the Beam Emission Spectroscopy (BES) diagnostic system. The goals are to visualize the spatial structure and time evolution of turbulent eddies, as well as to obtain the 2D statistical properties of turbulence. The measurements are obtained with an array of localized BES spatial channels configured to image a midplane region of the plasma. 32 channels have been deployed, each with a spatial resolution of about $1 \mathrm{~cm}$ in the radial and poloidal directions, thus providing measurements of turbulence in the wave number range $0<k_{\perp} \leqslant 3 \mathrm{~cm}^{-1}$. A 5 (radial) $\times 6$ (poloidal) channel grid provides time-resolved images near the outer midplane at the sampling frequency of $1 \mathrm{MHz}$, thus providing a modest spatial resolution, high throughput, high time resolution turbulence imaging system. The images and resulting movies have broad application to a wide variety of fundamental turbulence studies: imaging of the highly complex, nonlinear turbulent eddy interactions, measurement of the 2D correlation function, and $S\left(k_{r}, k_{\theta}\right)$ wave number spectra, and direct measurement of the equilibrium and time-dependent turbulence flow field. The time-dependent, two-dimensional turbulence velocity flow-field is obtained with time-delay-estimation techniques. (C) 2003 American Institute of Physics.
\end{abstract}

[DOI: $10.1063 / 1.1535248$ ]

\section{INTRODUCTION}

Measurements of fluctuations of key plasma parameters (e.g., density, temperature, potential) are essential to characterizing and more thoroughly understanding plasma turbulence and its associated anomalous transport, one of the central outstanding scientific issues in the fusion energy sciences. Turbulence arises from microinstabilities driven by density and temperature gradients inherent to magnetically confined plasmas. ${ }^{1}$ This turbulence is believed to be largely responsible for the high levels of cross-field radial transport of particles, energy and momentum observed in tokamak and other magnetic plasma configurations. As such, it is essential to obtain turbulence measurements to characterize, understand and mitigate this largely undesired turbulent transport, and ultimately to be able to predict the magnitude of such turbulent transport in future experimental fusion devices.

Turbulence in magnetically confined tokamak plasmas is a highly nonlinear and essentially two-dimensional phenomenon. It is manifest locally as fluctuations in such plasma parameters as density, temperature, electrostatic potential, magnetic field, and velocity. The confining magnetic field defines the relevant directions, and turbulent eddies are elongated along the confining magnetic field with correlation lengths in this toroidal direction comparable to the device scale $(\sim 1 \mathrm{~m})$, while correlation lengths perpendicular to the magnetic field are typically of order $5-10$ ion gyroradii $(\sim 1$ $\mathrm{cm})$. As such, it is critical to obtain two-dimensional mea-

\footnotetext{
${ }^{a}$ Current address: 13-367 General Atomics, P.O. Box 85608, San Diego, California 92186-5608.

${ }^{b)}$ Presently at CEA, Association Euratom-CEA, Cadarache, France.
}

surements of relevant fluctuating quantities perpendicular to the magnetic field. Most fluctuation measurements have focussed on zero and one-dimensional measurements as a result of the diagnostic systems or measurement technique. While such measurements have proven very valuable at characterizing turbulence in a wide variety of experimental devices and operational regimes, to make the next step to diagnosing the highly nonlinear properties of turbulence ${ }^{2,3}$ and rigorously comparing measurements to simulations and challenging models for turbulent transport, it has been recognized that two-dimensional fluctuation measurements will be an essential element of advancing turbulence physics.

Two-dimensional measurements of density fluctuations have broad application to a number of critical outstanding issues in turbulence research. These include direct visualization or imaging of turbulent eddy structures, measurement of the temporal dynamics of such eddies, radial shear in the poloidal velocity flow, equilibrium, and time-varying turbulent velocity dynamics, 2D correlation function and $S\left(k_{r}, k_{\theta}\right)$ wave number spectrum, and ultimately to quantify turbulence growth rates and energy cascades within the turbulent wave number spectrum. One pertinent example is the search for zonal flow signatures ${ }^{4}$ in the turbulence flow field. Such measurements require 2D fluctuation measurements to obtain the time-dependent and radially varying turbulence flowfield.

To obtain two-dimensional measurements of density fluctuations in the confined regions of hot plasmas, the Beam Emission Spectroscopy (BES) diagnostic system on the DIII-D tokamak (Ref. 13) has been deployed to obtain measurements in the radial and poloidal plane. ${ }^{5}$ These measure- 
ments have provided modest-spatial-resolution images of tokamak turbulence and been used to study turbulence flow and flow shear dynamics. ${ }^{6}$ These measurements will be discussed in detail. In parallel, new analysis techniques are being developed to more effectively exploit these new 2D measurements.

This paper will discuss the diagnostic system, examples of turbulence imaging measurements, and progress on development of new analysis techniques. We note that twodimensional turbulence images have been obtained in relatively cold plasmas using Langmuir probe arrays, ${ }^{7}$ and a gas puff imaging system has been recently developed to image light emission from edge/scrape-off layer turbulence in C-MOD and NSPX. ${ }^{8}$

\section{THE BES TURBULENCE IMAGING DIAGNOSTIC SYSTEM}

The BES diagnostic system measures local, longwavelength $\left(k_{\perp} \rho_{I}<1\right)$ density fluctuations by observing the fluorescence of a heating neutral beam. ${ }^{9,10}$ A DIII-D deuterium neutral beam, injected tangentially at $\sim 75 \mathrm{keV}$ to heat, fuel, and rotate the plasma, is collisionally excited via interactions with plasma electrons and ions. BES measures density fluctuations by observing the Doppler-shifted $D_{\alpha}$ emission $(n=3-2, \lambda \approx 653-655 \mathrm{~nm})$. Fluctuations in the light emission intensity are proportional to the local density fluctuations, $\widetilde{n} / n=C(\widetilde{I} / I)$, with a proportionality factor, $C$, that depends on local plasma density, temperature, beam energy and $Z_{\text {eff }}$. For typical DIII-D plasma parameters, $C \approx 2-3 .^{11}$

The optical viewing sightlines are deployed so that they are nearly tangent to a magnetic flux surface at the intersection point with the neutral beam volume to achieve good radial and poloidal resolution. Furthermore, the sightlines are angled by about $5^{\circ}$ to horizonal to approximately match the local magnetic field pitch angle since turbulent structures are expected to be aligned with a magnetic field line. The measurement technique is spatially-integrated whereby each spatial channel images a sampling volume defined by the intersection of the optical sightline and neutral beam. Each volume has an approximately $1 \mathrm{~cm}$ diam region in the radial and poloidal cross section, and is extended about $12 \mathrm{~cm}$ in the toroidal direction. This allows for a sensitivity to fluctuations in the range $k_{r}, k_{\theta}<3 \mathrm{~cm}^{-1}$, encompassing the range where long-wavelength turbulence associated with such instabilities as ion temperature gradient and trapped-electron modes are predicted to exist.

The BES system at DIII-D (Ref. 12) consists of 32 spatial channels. A high throughput optical system images light onto discrete fiber optic bundles. Light is collected with an $f=40 \mathrm{~cm}, f / 2$, objective lens that focusses light onto a bundle of 4 1-mm PCS silica fibers (N.A. $=0.25$ ) arranged in a square configuration. The fiber bundles efficiently relay the light to remotely located spectrometers. Each spectrometer consists of an $f / 2$ collimating lens, a $\Delta \lambda \approx 3 \mathrm{~nm}$ interference filter and an aspheric $f / 1$ lens to image the light onto a photodiode detector. Photodiode detectors and preamplifier electronics ${ }^{14}$ are cryogenically cooled to reduce dark current and electronic noise. Signals are passed through antialiasing

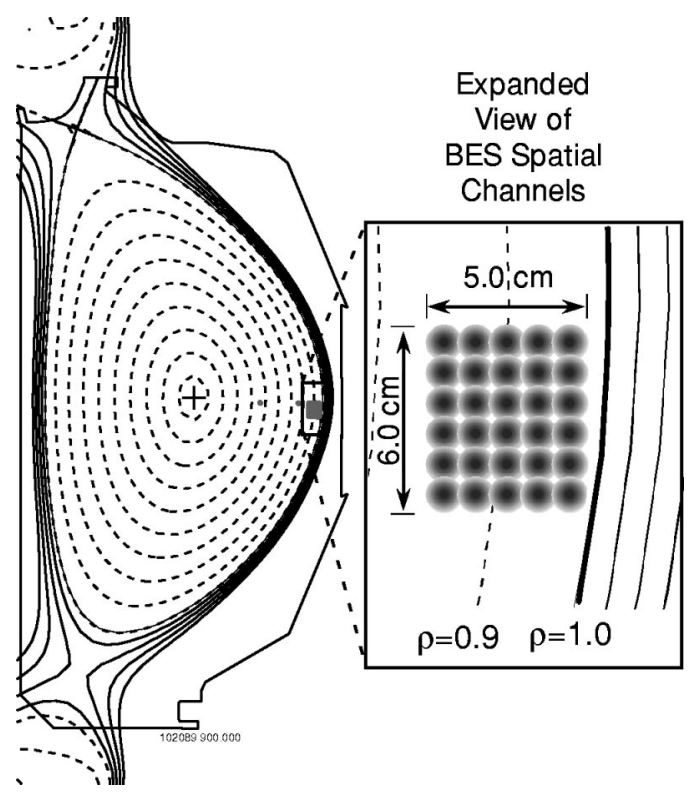

FIG. 1. BES channel arrangement for an imaging application. 30 discrete channels are deployed in a 5 (radial) $\times 6$ (poloidal) arrangement on the outer radial midplane.

circuits (cutoff at $f \approx 450 \mathrm{kHz}$ ) and digitized at $1 \mathrm{MHz}$ typically with a $0.5 \mathrm{~s}$ time record.

\section{TURBULENCE IMAGES AND MOVIES}

For imaging applications, the 32 spatial channels are deployed in a rectangular grid. A flexible mounting array allows for deployment in a variety of $2 \mathrm{D}$ configurations. A typical channel deployment would consist of a grid with 5 channels radially by 6 poloidally $(5 \times 6)$. Each channel is separated by about $1 \mathrm{~cm}$ in the radial and poloidal direction, thus covering a $5 \times 6 \mathrm{~cm}$ region in the plasma. Alternative deployments might include $4 \times 8$ or $6 \times 5$ arrangement. Nonrectangular arrangements are routinely deployed for specific experiments where additional radial or poloidal coverage may be needed. A typical channel arrangement is shown in Fig. 1 overlaid with a magnetic equilibrium reconstruction. The channels are shown with their relative sizes in the radial and poloidal plane with the shading qualitatively representing the magnitude of the spatial transfer function in these dimensions. The fiber mounting array can be radially scanned on a shot-to-shot basis with a remotely controlled motorized scanner.

The BES system deployed in this fashion provides 2D measurements of density fluctuations and so can be considered a modest spatial resolution, very high throughput, high sensitivity, high time resolution imaging system. Measurements can be ensemble averaged in time to assess the spectral properties of the turbulence (power spectra, radial and poloidal correlations, wave number spectra, etc.). Timeresolved measurements of turbulence can be obtained when the turbulence is of sufficient amplitude so that the instantaneous density fluctuation signals exceed the inherent photon noise and electronic noise in the detector system. This is typically achieved when the normalized rms density fluctuations, $\widetilde{n} / n$, are greater than roughly $5 \%$. This is often the 

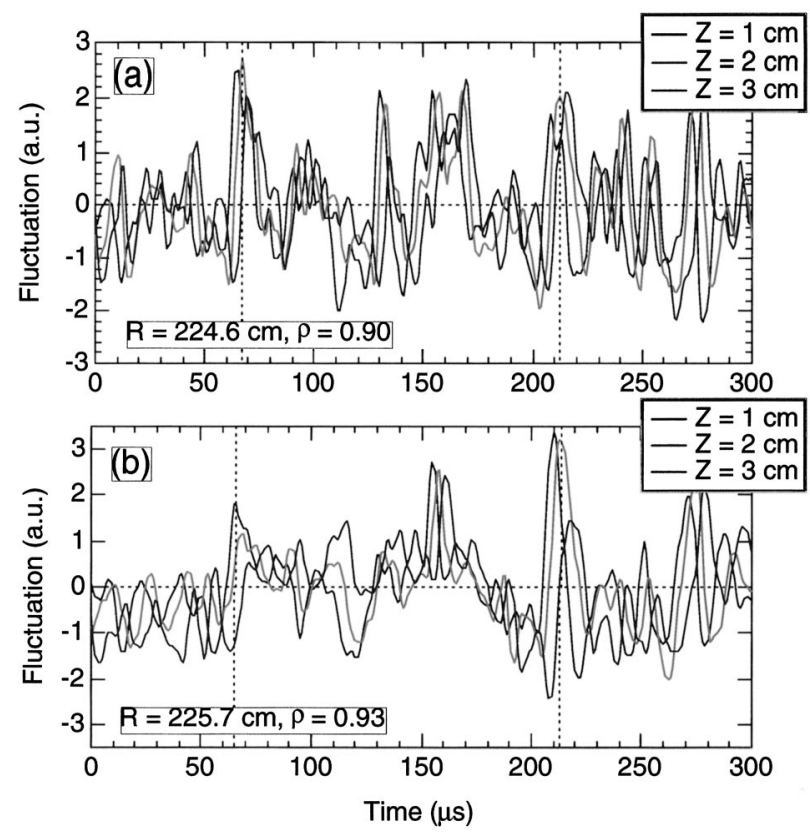

FIG. 2. Time-series fluctuation data acquired at $1 \mathrm{MHz}$ near outer region of an $L$-mode discharge, (a) three poloidally-separated channels at $\rho=0.90, Z$ $=1,2,3 \mathrm{~cm}$, (b) three poloidally-separated channels at $\rho=0.93, Z=1,2,3$ $\mathrm{cm}$, showing high coherence and poloidal advection of eddy structures.

case in the outer regions of $L$-mode plasmas, $\rho>0.85$ ( $\rho$ is the normalized toroidal flux radial coordinate). Figure 2 shows an example time series of fluctuation data at poloidally displaced channels demonstrating the high coherence of the signals and the few microsecond temporal offset resulting from poloidal advection of the turbulent eddies.

Movies of turbulence are assembled from the discrete spatial measurements through straightforward image processing methods. The time signals from individual spatial channels are first frequency-filtered over the spectral range where the broadband turbulence is observed, typically $2 \mathrm{kHz}<f<200 \mathrm{kHz}$ for the outer regions of the plasma discussed here. This removes excess photon and electronic noise at higher frequencies and beam power supply oscillations at very low frequencies. For each time point, the discrete signals are assembled into the 2D grid and then fitted with a two-dimensional spline calculated as a minimum curvature surface. For a typical deployment, 30 channels are arranged in a 5 radial $\times 6$ poloidal grid and the $2 \mathrm{D}$ spline yields a $50 \times 60$ pixel smoothed surface. This data is then linearly interpolated to the desired image size, e.g., 250 $\times 350$ pixels. The images are displayed with a false-color mapping. Contour mapping is utilized to visually facilitate a more quantitative interpretation of the images and movies. A set of frames from an example turbulence movie is shown in Figs. 3(a) $-3(\mathrm{f})$. Here each image is separated in time by 8 $\mu \mathrm{s}$.

Individual images or frames of density fluctuations are helpful for visualizing the size and shape of turbulent eddy structures, but the scientific value is greatly enhanced via the long time sequence of such images, assembled into movies. The temporal dynamics elucidate the complex and highly nonlinear interactions of the turbulent eddies and their behavior on the confined and open magnetic flux surfaces. The time-averaged turbulence flow velocity and radial shear in this velocity are readily apparent, but it is also clear that the transient flow dynamics deviate significantly from the timeaveraged or ensemble-averaged statistical properties. It is possible, for example, to visualize eddies being stretched and torn apart as a result of the radially-sheared poloidal flow. This is consistent with the model of $E \times B$ shear stabilization of turbulence that has been successfully invoked to explain improved confinement in a wide variety of plasma operational modes. ${ }^{15}$

These time sequences of density fluctuation images have also allowed for the direct visualization of turbulent crossfield radial particle transport in confined plasmas. In regions near the plasma edge, it is particularly evident that there is an asymmetry in the radial trajectories of positive and negative density fluctuations. Positive density fluctuations tend to drift outward radially, while negative fluctuations (density "holes") tend to drift inward. This can be seen in the sequence of images in Fig. 3. A "red" (positive) eddy (top center) is initially seen poloidally offset from but radially aligned with the "blue" (negative) eddy (center). As time progresses, the two-eddy system appears to rotate in such a way that the positive eddy drifts radially outward (to the right) and the negative eddy radially inward (left), thus qualitatively showing a net outward radial flux of particles. It is noted that this is consistent with a model of radial transport by electrostatic drift waves ${ }^{16}$ in which there is a finite (nonzero) phase shift between the (measured) density and the (unseen) potential fluctuations. This finite phase shift is required to yield net radial transport since a zero phase shift would result in the same quantity of inward and outward flux for no net transport. Since fluctuation measurements obtained with BES are calibrated and measure $\widetilde{n} / n$, the quantitative radial flux can be estimated; this will be the subject of a future study.

In addition, a modest shearing is evident from the background equilibrium poloidal flow in Fig. 3. This flow is upward at all locations, but has increased magnitude at smaller radii (left side). This results in a tendency for eddies to drift upward and be stretched toward the upper left. Also, an accretion of two "red" eddies is evident in the third to fifth frames as the initially distinct eddy structures (third frame) join together as a radially extended eddy in the fourth frame which becomes more symmetric by the fifth frame.

\section{WAVE NUMBER SPECTRA}

The first-order ensemble-averaged statistical correlation properties of turbulence (correlation lengths, decorrelation times, wave number spectra) are relevant for comparing measurements with nonlinear turbulence simulations. ${ }^{17}$ Past studies have typically measured the 1D correlation properties and wave number spectra, $S\left(k_{r}\right)$ and $S\left(k_{\theta}\right) .{ }^{18}$ The 2D measurements allow for determination of the full twodimensional density fluctuation wave number spectra, $S\left(k_{r}, k_{\theta}\right)$. Given the inherently two-dimensional nature of tokamak turbulence, this is a quantity of particular interest for comparisons to predictions and simulations.

The wave number spectrum is obtained here from mea- 

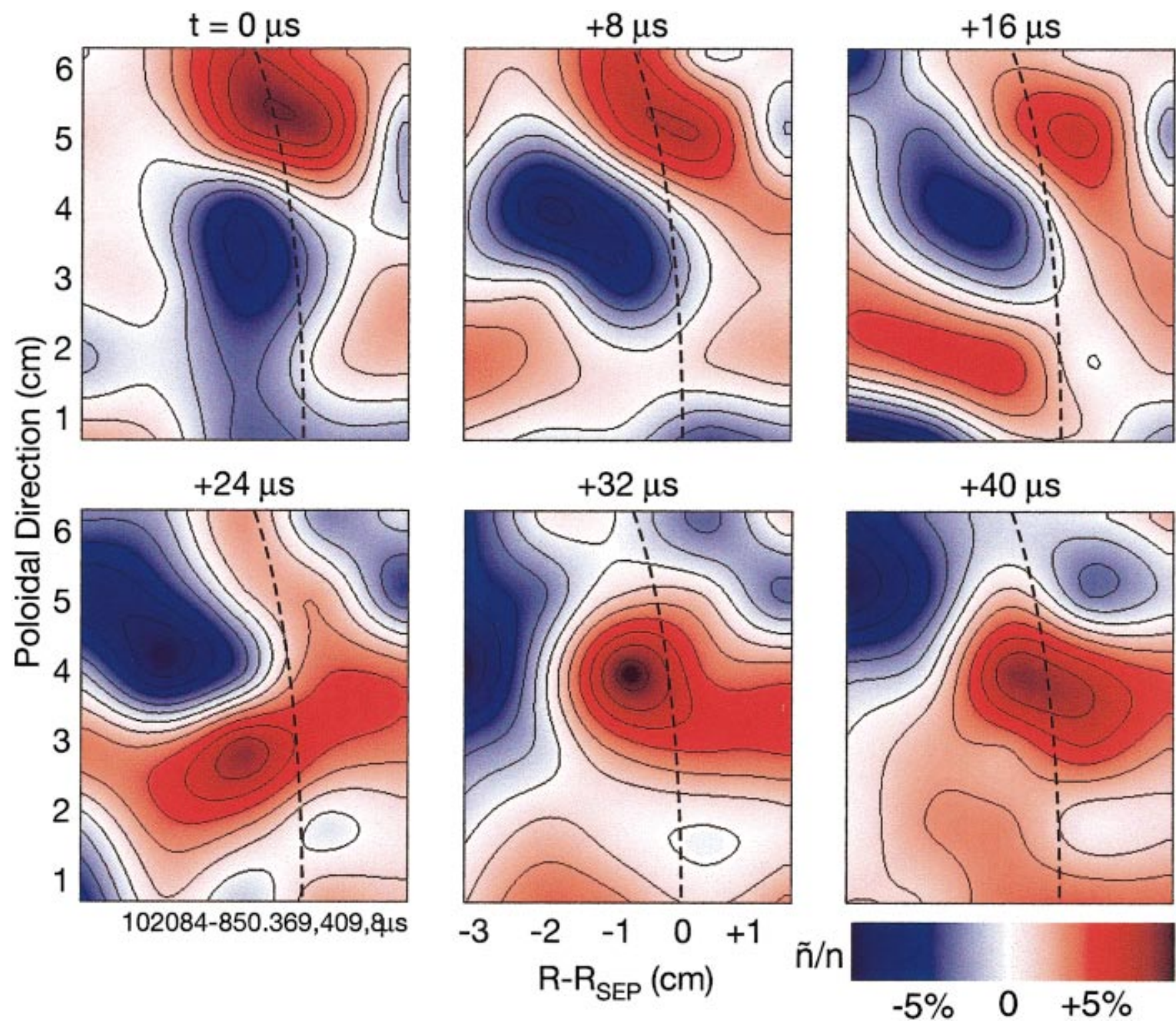

FIG. 3. (Color) Series of six sequential images of turbulence, each separated by $8 \mu$ s. Images obtained via $2 \mathrm{D}$ spline to $5 \times 6$ channel grid. Red indicates a positive density fluctuation, blue indicates a negative density fluctuation about the equilibrium density (white). Contour lines indicate relative fluctuation magnitude. Dashed line represents the magnetic separatrix.

surement of the two-point correlations across a $2 \mathrm{D}$ array of data. Pairwise correlation functions are used to construct the 2D correlation function, $\rho(\Delta r, \Delta z, t=0)$. A sample correlation function is shown in Fig. 4(a). Those data were obtained in an $L$-mode plasma at $\rho=0.85-1.0$. The $S\left(k_{r}, k_{\theta}\right)$ spectra is then obtained from this correlation function with a $2 \mathrm{D}$ spatial transform using the discrete cosine transform (DCT), which is also commonly used for image compression. The DCT transforms a real vector or array into another real vector or array, unlike the FFT which yields a complex output. While both the DCT and 2D FFT are complete transforms and can be inverted back to obtain the original data, the DCT offers an advantage of higher wavenumber resolution and so is used here (Fig. 4).

The 2D correlation function [Fig. 4(a)] shows strong asymmetry in the radial and poloidal directions, with a monotonically decaying correlation in the radial direction, while the poloidal correlation exhibits a decaying wavelike structure. This is consistent with results obtained with 1D measurements in the radial or poloidal directions separately. ${ }^{18}$ The corresponding 2D wave number spectrum is shown in Fig. 4(b). This shows a clear peak in the spectrum near $k_{r}=0$ and $k_{\theta} \approx 1 \mathrm{~cm}^{-1}$.

\section{VELOCITY FLOW FIELD}

The magnitude and direction of the turbulence flow-field enables measurement of turbulence shearing, mode identification, as well as nonlinear turbulence properties such as zonal flows and energy cascades. The turbulence flow field is here defined as the local average flow direction of turbulent eddies, or the turbulence group velocity, and should be distinguished from the bulk mass flow of the plasma itself. The turbulence velocity is believed to be a function of local $E_{r}$ $\times B_{T}$ velocity and some fraction of the ion or electron diamagnetic velocity. 
The flow-field can be measured as an ensemble-averaged property of the turbulence, or, if the signal to noise is sufficient, measured in a time-dependent fashion on time scales up to that of the density fluctuation spectrum itself. An ensemble-averaged flow-field can be determined via a calculation of the two-point time-lag cross correlations. ${ }^{8}$ The timedelay correlation function, a sample of which is shown in Fig. 5(a), is calculated by integrating over a specified frequency range. The envelope of the correlation function is calculated using the Hilbert transform of the correlation. The local velocity is then obtained simply as the known channel separation divided by the time at the centroid of the cross correlation envelope. It is generally observed that there is no steady-state radial eddy velocity, but the steady-state poloidal velocities are significant and can be of order of several $\mathrm{km} / \mathrm{s}$. A typical turbulence-flow field is shown in Fig. 5(b) with the arrows pointing in the flow direction (poloidal) and with a length scaled to the average flow magnitude. In this particular case, it is observed that the flow reverses locally. In edge regions of the plasma, as shown here, the turbulence can be highly dispersive, i.e., the velocity can depend strongly on the wave number or frequency band being considered. Thus it may be necessary to specify the frequency or wave number range for a given flow field.

The time-dependent turbulence flow field is of interest for several applications, including determination of the turbulent $E \times B$ flux ${ }^{19}$ and self-regulating zonal flows. Two approaches have been developed to ascertain the timedependent flux on turbulence-relevant time scales. One method uses a wavelet-based cross-correlation technique to determine the time-dependent phase relationship between two time series. This has been described in Ref. 19.

An alternate technique to obtain time-dependent time delay estimates utilizes a short time-window cross-correlation method. This technique is conceptually similar to the timeaveraged method described above, but rather than performing an ensemble-averaged cross-correlation, the correlation is obtained over a short time-window. Here, the data is oversampled in time to achieve subsampling time resolution. A time window is chosen appropriately for the required timeresolution satisfying the Nyquist criteria. A time-lag crosscorrelation is performed between the two oversampled time series fluctuation data within the selected time window. The time delay corresponding to the peak of the cross-correlation is recorded. A time series of peak time delays is thus constructed by scanning the data. A Hanning window is applied to the input time series data prior to the cross-correlation analysis to avoid spurious spectral features in the resulting time or velocity data set. Standard spectral analysis techniques are then applied to the resulting data to examine flow properties and characteristics.

An example of the spectrum of data analyzed with this method is shown in Fig. 6. Here the poloidal flow velocity was measured at two poloidally separated locations $(\Delta Z$ $=5 \mathrm{~cm}$ ). The cross power between these two TDE measurements shows a clear coherent peak near $15 \mathrm{kHz}$ in the poloidal flow velocity. Detailed analysis of this phenomenon and its spatial structure is discussed in Ref. 20.

It has been found that the wavelet TDE technique
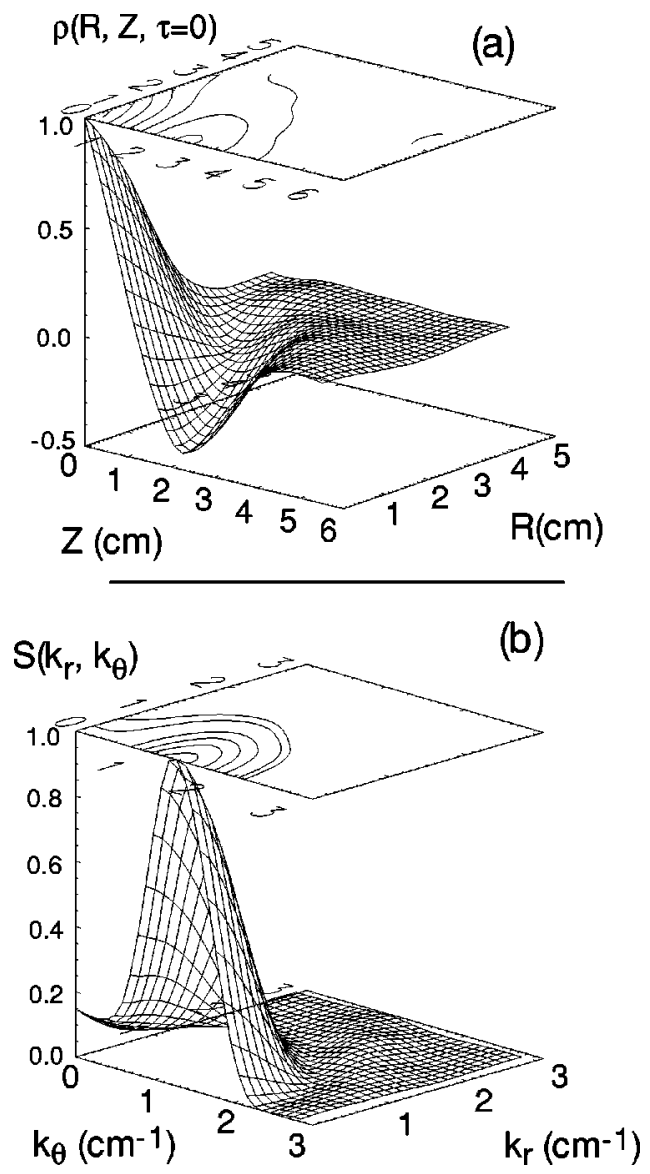

FIG. 4. (a) Two-dimensional ( $\Delta t=0)$ spatial correlation function, and (b) the corresponding 2D wave number spectrum, $S\left(k_{r}, k_{\theta}\right)$.

achieves higher frequency resolution, while the short timewindow cross-correlation method generally results in a higher signal quality for lower frequency phenomena.

\section{FUTURE DIAGNOSTIC DEVELOPMENTS}

Initial turbulence imaging measurements obtained with the BES system have been useful for discerning the timeevolving and ensemble-averaged 2D turbulence characteristics. To further extend the diagnostic technique and apply these measurements to a broader range of plasma conditions and spatial regions, it will be highly desirable to increase the sensitivity of the measurement as well as to expand the spatial domain being sampled. The present BES system, limited by channel number, images a spatial region that is about two to three turbulence correlation lengths in domain size. A next generation density fluctuation system is being designed that should have nearly ten times the sensitivity of the existing system and will provide up to an additional 32 highsensitivity spatial channels.

The sensitivity will be enhanced through measurement of additional spectral signals, increasing the spatial sampling area, utilizing a higher throughput optical system, and increasing the length of the data time record. Density fluctuations are currently measured with BES by observing the Doppler-shifted $D_{\alpha}$ emission from the collisionally excited beams. The next-generation system will observe this emission as well as the thermal charge exchange emission arising 
(a)

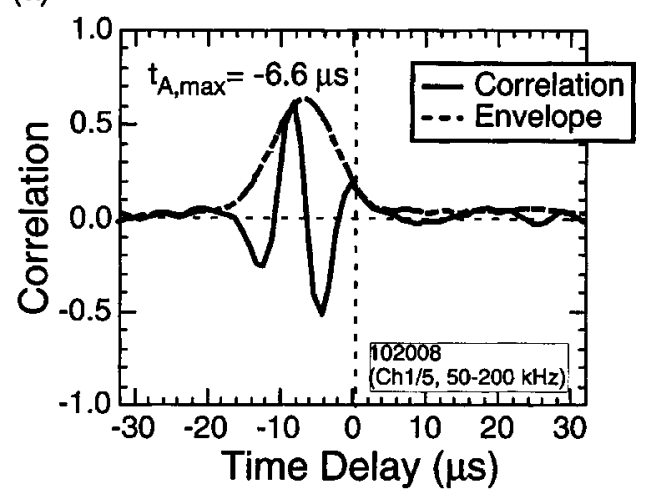

(b)

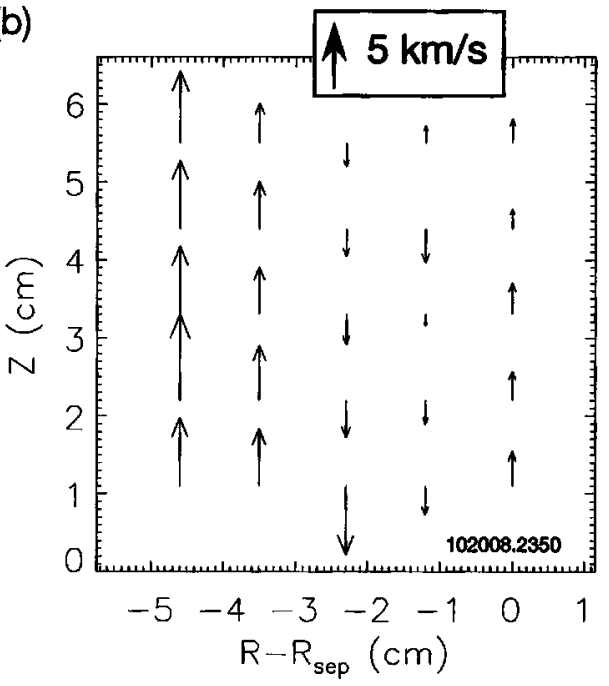

FIG. 5. (a) A typical ensemble-averaged time-lag cross-correlation between poloidally separated channels (correlation shown as solid line, envelope as dashed line) showing the peak correlation near $-7 \mu$ s, indicative of the equilibrium poloidal flow. (b) The time-averaged turbulence velocity flow field, obtained from two-point cross-correlations across a $5 \times 6 \mathrm{~cm}$ grid of data.

from charge exchange reactions between beam atoms and the background deuterium ions. This thermally broadened emission, centered near $\lambda_{o}=656.1 \mathrm{~nm}$, will be spectrally isolated from the intense but narrow edge $D_{\alpha}$ emission utilizing a high throughput spectrometer. The specialized spectrometer will utilize custom-designed near unity transmission gratings (Kaiser Optical Systems, Inc.) optimized for unpolarized light transmission at high dispersion at the selected wavelength band. The optical throughput will be increased by more optimally aligning the aspect ratio of the image spotsize in the plasma to the inherent turbulence structures, which typically have longer poloidal correlation lengths than radial correlation lengths. The present image sizes are radially and poloidally symmetric.

These systematic improvements are expected to provide a net enhancement of signal strength of nearly an order of magnitude, resulting from additional observed spectral emis-

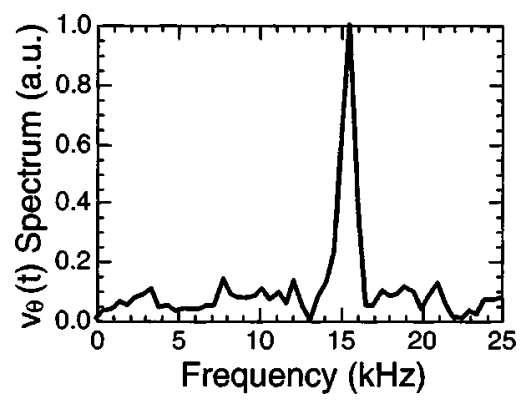

FIG. 6. Cross-power spectrum of the time-dependent poloidal flow field obtained using a short-time-window cross-correlation method.

sions $(* 2)$, increased image size $(* 2.5)$, and increased optical efficiency $(* 1.5)$. In addition, an increased time record will allow for improved signal to noise in the ensemble-averaged spectral analysis. This series of enhancements should facilitate further investigation of two-dimensional turbulence behavior in high confinement regimes and in the core confinement region.

\section{ACKNOWLEDGMENTS}

The authors would like to thank the DIII-D program for its support of the diagnostic development program and for experimental run time. The research performed under U.S. DOE Grant No. DE-FG03-96ER54373 and Contract No. DE-AC03-99ER54463.

${ }^{1}$ B. A. Carreras, IEEE Trans. Plasma Sci. 25, 1281 (1997).

${ }^{2}$ J. S. Kim, R. D. Durst, R. J. Fonck, E. Fernandez, A. Ware, and P. W. Terry, Phys. Plasmas 3, 3998 (1996).

${ }^{3}$ J. S. Kim, R. J. Fonck, R. D. Durst, E. Fernandez, P. W. Terry, S. F. Paul, and M. C. Zarnstorff, Phys. Rev. Lett. 79, 841 (1997).

${ }^{4}$ T. S. Hahm, K. H. Burrell, Z. Lin, R. Nazikian, and E. J. Synakowski, Plasma Phys. Controlled Fusion 42, A205 (2000).

${ }^{5}$ C. Fenzi, R. J. Fonck, M. Jakubowski, and G. R. McKee, Rev. Sci. Instrum. 72, 988 (2001).

${ }^{6}$ G. R. McKee, C. Fenzi, and R. J. Fonck, IEEE Trans. Plasma Sci. 30, 62 (2002).

${ }^{7}$ S. J. Zweben and R. W. Gould, Nucl. Fusion 25, 171 (1985).

${ }^{8}$ S. J. Zweben, et al., Phys. Plasmas 9, 1981 (2002).

${ }^{9}$ R. D. Durst, R. J. Fonck, G. Cosby, and H. Evensen, Rev. Sci. Instrum. 63, 4907 (1992).

${ }^{10}$ R. J. Fonck, P. A. Duperrex, and S. F. Paul, Rev. Sci. Instrum. 61, 3487 (1990).

${ }^{11}$ T. A. Gianakon, R. J. Fonck, J. D. Callen, R. D. Durst, and J. S. Kim, Rev. Sci. Instrum. 63, 4931 (1992).

${ }^{12}$ G. McKee, R. Ashley, R. Durst, R. Fonck, M. Jakubowski, K. Tritz, K. Burrell, C. Greenfield, and J. Robinson, Rev. Sci. Instrum. 70, 913 (1999).

${ }^{13}$ J. L. Luxon, Nucl. Fusion 42, 614 (2002).

${ }^{14}$ R. J. Fonck, R. Ashley, R. Durst, S. F. Paul, and G. Renda, Rev. Sci. Instrum. 63, 4924 (1992).

${ }^{15}$ K. H. Burrell, Science 281, 1816 (1998); Phys. Plasmas 4, 1499 (1997).

${ }^{16}$ W. Horton, Rev. Mod. Phys. 71, 735 (1999).

${ }^{17}$ D. W. Ross, R. V. Bravenec, W. Dorland et al., Phys. Plasmas 9, 177 (2002).

${ }^{18}$ R. J. Fonck, G. Cosby, R. D. Durst et al., Phys. Rev. Lett. 70, 3736 (1993).

${ }^{19}$ M. Jakubowski, R. J. Fonck, C. Fenzi, and G. R. McKee, Rev. Sci. Instrum. 72, 996 (2001).

${ }^{20}$ M. Jakubowski, R. J. Fonck, and G. R. McKee, Phys. Rev. Lett. 89, 265003 (2002). 